\title{
The disaster worker resiliency training program: a randomized clinical trial
}

\author{
Brittain L. Mahaffey ${ }^{1,2}$ (1) Daniel M. Mackin ${ }^{3} \cdot$ Jonathan Rosen $^{4,5} \cdot$ Rebecca M. Schwartz $^{2,6,7,8} \cdot$ Emanuela Taioli $^{2,6}$. \\ Adam Gonzalez ${ }^{1,2}$
}

Received: 4 February 2020 / Accepted: 9 May 2020 / Published online: 24 May 2020

○) Springer-Verlag GmbH Germany, part of Springer Nature 2020

\begin{abstract}
Objectives Disaster workers are at elevated risk for mental health problems as a result of trauma exposures during response efforts. One possible way to prevent mental health problems is to build-up coping resources that promote resilience to the effects of disaster work. The primary aim of this study was to evaluate the efficacy of a resilience building workshop, the Disaster Worker Resiliency Training Program (DWRT), in disaster workers previously exposed to Hurricane Sandy.

Methods Disaster workers $(N=167)$ were randomly assigned to the DWRT workshop $(n=78)$ or a waitlist $(n=89)$. Workers completed self-report measures on healthy lifestyle behaviors, perceived stress, depression, and Posttraumatic Stress Disorder (PTSD) symptoms at baseline and 3-month follow-up. They also completed a measure assessing subsequent trauma-exposure between the baseline and 3-month post-intervention.

Results Participants in the workshop condition, as compared to those in a waitlist control, reported significantly greater improvements from pre-intervention (T1) to 3-month follow-up (T2) in healthy lifestyle behaviors $\left(\eta^{2}=.03 ; p=.03\right)$, stress management $\left(\eta^{2}=.03, p=.04\right)$, and spiritual growth $\left(\eta^{2}=.03, p=.02\right)$. Among participants reporting subsequent trauma exposures between T1 and T2 $(n=101)$, participants in the waitlist condition, were more likely to report significant increases in perceived stress $\left(\eta^{2}=.07, p<.01\right)$, PTSD $\left(\eta^{2}=.05, p=.03\right)$, and depression $\left(\eta^{2}=.07, p<.01\right)$ symptoms.

Conclusions Participation in the resilience workshop promoted engagement in positive health behaviors and reduced the incidence of mental health symptoms, especially when administered prior to a repeat trauma exposure. Further research is needed to evaluate the long-term health effects of participation in the program.
\end{abstract}

Keywords Trauma $\cdot$ PTSD $\cdot$ Health behavior $\cdot$ Resilience $\cdot$ Intervention

Brittain L. Mahaffey

Brittain.Mahaffey@stonybrookmedicine.edu

1 Department of Psychiatry \& Behavioral Health, Stony Brook University School of Medicine, Putnam Hall-South Campus, Stony Brook, NY 11794, USA

2 Center for Disaster Health Trauma, \& Resilience at Stony Brook University, Mount Sinai, and Northwell Health, Great Neck, NY, USA

3 Department of Psychology, Stony Brook University, Stony Brook, NY, USA

4 National Clearinghouse for Worker Safety \& Health Training, Washington, DC, USA
5 AJ Rosen \& Associates LLC, New York, USA

6 Department of Population Health Science and Policy and Institute of Translational Epidemiology, The Icahn School of Medicine at Mount Sinai, New York, NY, USA

7 Occupational Medicine Epidemiology and Prevention, Northwell Health, Great Neck, NY, USA

8 Department of Occupational Medicine Epidemiology and Prevention, Zucker School of Medicine at Hofstra/Northwell, Great Neck, NY, USA 


\section{Introduction}

Disaster workers are often exposed to traumatic psychological experiences during disaster response efforts. As such, disaster responders are at increased risk for physical and mental health problems (Benedek et al. 2007; Berger et al. 2012). Depression and posttraumatic stress disorder (PTSD) are the most common mental health sequelae experienced by disaster workers (Bromet et al. 2017a, b; Galea et al. 2005; Neria et al. 2008; Thormar et al. 2010). Trauma-related mental health symptoms are also associated with increased engagement in negative health behaviors such as smoking, alcohol and drug abuse, as well as overall increased risk for physical morbidity and mortality (Thormar et al. 2010; Bruce et al. 1994; Lavretsky et al. 2002). Moreover, repeated trauma exposures, such as those experienced by professional responders (e.g., police, firefighters, and paramedics), increase the likelihood that workers will develop psychiatric symptoms (Neria et al. 2008; Breslau et al. 2008; Bromet et al. 2017a, b; Brunet et al. 2001; Caramanica et al. 2015; Shrira et al. 2014).

Despite the evidence of risk associated with repeated trauma exposures, most previous work in the area of disaster mental health has focused on efforts to prevent the onset of PTSD via acute post-trauma interventions such as Critical Incident Stress Management (CISM). CISM includes pre-incident training, initial post-incident defusing, group debriefing (e.g., Critical Incident Stress Debriefing), and further counseling (Mitchell and Everly 1997a). CISM is an umbrella term for post-trauma interventions, which are often heterogeneous in terms of content and delivery (e.g., varying numbers of sessions). While individuals who participate in such programs report finding them subjectively helpful for processing traumatic exposures (Irving and Long 2001; Mitchell and Everly 1997b), there have been few high quality randomized clinical trials (RCTs) and there is some evidence suggesting that aspects of these interventions (i.e., psychological debriefing) may inadvertently exacerbate some participants' distress (Tuckey and Scott 2014). Psychological First Aid (PFA) is an alternative intervention intended to be responsive to some of the limitations of CISD. PFA focuses on managing acute stress symptoms and deemphasizes debriefing strategies (Nash and Watson 2012; Ruzek et al. 2007). Nonetheless, the literature supporting PFA also remains sparse with few methodologically strong randomized trials (Fox et al. 2012). Moreover, neither intervention focuses on building up coping resources in advance of a traumatic exposures in order to promote psychological resilience (Davydov et al. 2010).

Enhancing resilience has been identified as being integrally important for mitigating the effects of disasters
(White House 2007). Resilience in this context is conceptualized as the adaptive use of coping skills such as utilizing social supports, engaging in self-care activities, and improving health behaviors. Improving resilience in vulnerable communities, such as those previously affected by large-scale disasters, and those likely to be called upon repeatedly to respond to disasters (e.g., Red Cross/FEMA personnel, police, firefighters, and paramedics) is particularly important. For example, many World Trade Center (WTC) responders were either personally impacted and/ or called upon as part of their job to respond to Hurricane Sandy in 2012. Evidence suggests that the subsequent exposure to Hurricane Sandy substantially increased the likelihood of PTSD and Major Depressive Disorder (MDD) in this population (Bromet et al. 2017a, b). Thus, there is a critical need for resilience building programs aimed at assisting disaster workers in the development of adaptive coping skills. Such programming is essential for maintaining a resilient disaster response workforce (White House 2007).

Several empirically supported programs have been developed to enhance resilience in people with psychological and/or physical health problems (e.g., the Relaxation Response Resiliency Program [3RP] (Park et al. 2013); Mindfulness-Based Stress Reduction [MBSR] (KabatZinn 2003). These programs are effective in reducing perceived stress, improving quality of life, and attenuating mood and anxiety symptoms (e.g., Sood et al. 2011). However, they generally require a significant time commitment (i.e., 8-12 weeks), can be costly to deliver, and are not designed specifically to address the types of stressors experienced by disaster workers. Disaster response agencies have responded to these shortcomings by developing training guides or brief disaster preparedness programs for their workforces, but often the criteria for the development of these programs is unknown and no data are publicly available on their efficacy.

The Disaster Worker Resiliency Training Program [DWRT; (National Institute 2012)] is a one-session (4-h), interactive resilience-training workshop developed by the National Institute of Environmental Health Sciences (NIEHS) and the Substance Abuse and Mental Health Services Administration (SAMHSA). The program was created via a multiphase process involving community input, a comprehensive literature review, and consultation with behavioral health experts. While traditional training programs for responders focus almost exclusively on psychoeducation and emotional support, the DWRT incorporates empirically supported psychological techniques such as motivational interviewing, health behavior goal setting, and relaxation training into its curriculum. The program teaches strategies for recognizing the signs and symptoms of traumatic stress reactions in oneself and others and normalizes help seeking 
behavior. It also encourages participants to think about ways to make themselves and their organizations more resilient to stress in advance of a crisis.

The goal of this study was to evaluate the efficacy of the DWRT program for promoting resilience and preventing the development of mental health symptoms in a sample of active disaster workers who responded to Hurricane Sandy. This study fills a critical gap in the disaster mental health literature by rigorously evaluating a brief and costeffective structured program designed to promote resilience for disaster workers via a randomized clinical trial (RCT). We hypothesized that, relative to a waitlist control group, the participants receiving the DWRT program would have significant increases in their levels of adaptive health behaviors and levels of posttraumatic growth, and significantly decreased levels of perceived stress from baseline to followup at 3-months post-intervention. In the subset of participants exposed to subsequent trauma(s) between baseline and follow up, we also expect to also see lower levels of PTSD and depression symptoms in among the DWRT group, relative to those in the waitlist condition.

\section{Methods}

\section{Participants}

Participants included 167 disaster responders $\left(n_{\text {DWRT }}=78\right.$, $\left.n_{\text {waitlist }}=89 ; M_{\text {age }}=51.26, \mathrm{SD}=15.49\right)$ recruited from the Long Island and New York metropolitan area. Participants were recruited between March 1st, 2015 and February 27th, 2016 from 8 local agencies including the World Trade Center Health Program, United Way, and New York City Medical Reserve Corps. Hurricane Sandy responders were targeted for participation in this trial given their exposure to a common stressor and the fact that most reported continuing to be active in community disaster response efforts. Individuals who indicated they were no longer active disaster workers were excluded from the present trial given that the aim of the present trial was building resilience in an active disaster worker population. The sample was predominately Caucasian (69.9\%) and non-Hispanic $(86.7 \%)$, but was diverse in terms of gender ( $56.0 \%$ female), marital status (47.0\% married), and employment status $(50.6 \%$ full-time employment). Eight participants (6 waitlist, 2 DWRT) did not complete the follow-up assessment and were excluded from analyses (see Fig. 1 for consort flow diagram). Attritors did not differ from completers on any relevant demographic variables $(p$ 's $>.43)$.

\section{Procedure}

Participants were recruited for the study through organizations in the area where employees or volunteers are typically mobilized in disaster response efforts (e.g., ambulance services, local fire and law enforcement departments, hospitals, union groups, and medical centers). An initial stakeholder meeting was held at the sponsor institution, prior to beginning recruitment to increase community engagement with the project. Agency heads and community leaders were invited to share about their organizational training needs and were provided with an overview of the DWRT program. Additional recruitment methods included mailing flyers to agencies involved in hurricane response efforts and spitball recruitment (i.e., asking agency leaders to share information with their contacts in the responder community). Organizations were required to have approximately 20 eligible volunteers or staff members in order to participate. This was intended to ensure sufficient participation for randomization within-agency. Participation was strictly voluntary and held outside of regular business hours. Eligibility criteria included participation as a responder to the Hurricane Sandy disaster (professionally or as a volunteer) and active responder status. Eligibility was established through the completion of a phone screen and participants were required to provide informed written consent. This study was approved by the local university institutional review board.

All participants $(N=167)$ completed an initial online assessment (T1) including self-report surveys regarding their current healthy lifestyle behaviors, levels of stress, and symptoms of psychopathology. Participants were then randomized to either the DWRT workshop $(N=78)$ or a waitlist control condition $(N=89)$. Randomization was done within agency cohorts, rather than across the whole sample, in order to account for the clustering of volunteer versus professional responders within organizations. The DWRT workshop was administered by trained mental health professionals (clinical psychologist or social worker). Following the workshop, participants completed a short exit survey to assess satisfaction with the training. Symptom measures were not repeated immediately postintervention as no immediate change in anxiety, stress, or trauma symptoms was expected. Change in symptoms was assessed 3-months following completion of the workshop (T2) to enable time for participants to begin engaging in prescribed resilience building activities to allow time for subsequent work-related trauma and stress exposure. At $\mathrm{T} 2$, participants repeated the baseline measures as well as an additional questionnaire about subsequent exposure to traumatic events since the last assessment. All participants were compensated up to $\$ 60$ for their time and those assigned to the waitlist were invited to participate in the 


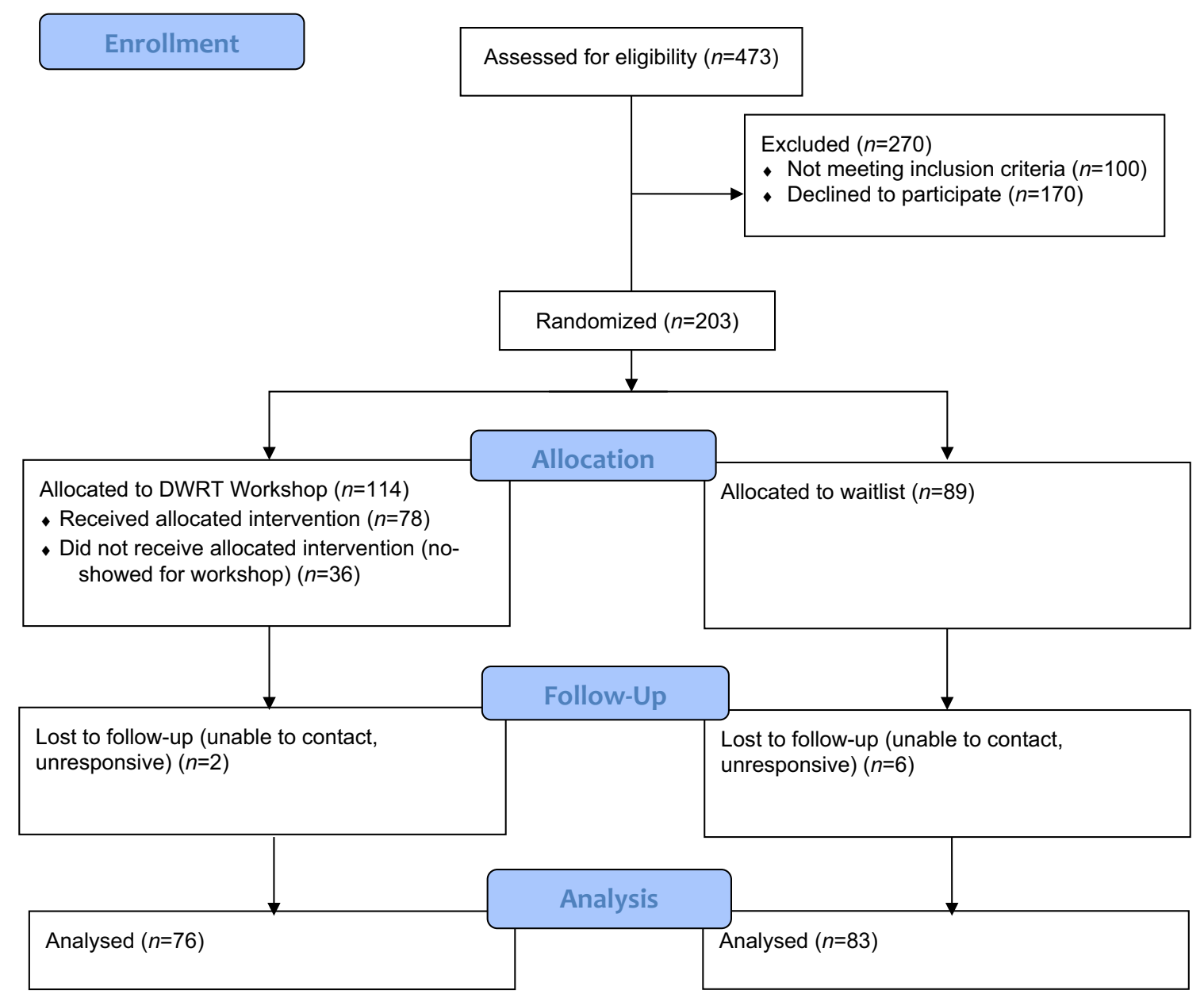

Fig. 1 CONSORT flow diagram for study participants. Participants lost to follow-up were included in baseline analysis only

workshop after the completion of the 3-month post-intervention period.

\section{Intervention}

\section{The Disaster Worker Resilience Training (DWRT) Program}

This 4-h workshop consists of a participant training manual, an instructor training manual, and a digital presentation. It uses adult training techniques that emphasize active participation in individual and group experiential learning activities. The curriculum is organized into a preface and four chapters, each with action-oriented learning objectives. Overall program objectives include demonstrating an ability to: (1) Recognize signs and symptoms of disaster work-related stress, (2) Obtain support through employer and community resources, and (3) Build resilience by using stress reduction and coping strategies. See Table 1 for further details on session content.

Sections providing psychoeducation on the effects of disasters were revised to include information on respiratory symptoms due to mold, asbestos and silica dust exposures during clean-up (NYS-DOH 2012) and issues related to mental and physical health comorbidity. In addition, emergency response personnel are a distinct group that differs from the general population. They have unique concerns (e.g., about privacy for police officers, as psychiatric problems may disqualify them from active duty), needs (e.g., to maintain their roles as protectors), and motivations (e.g., to give and to help others). To be maximally acceptable to responders and to take advantage of their unique strengths, the protocol was modified to account for the culture of this community.

\section{Measures}

\section{Health-promoting lifestyle profile II (HPLP II)}

The HPLP II (Walker et al. 1995) is a 52 item self-report questionnaire that measures the extent to which participants currently engage in healthy lifestyle behaviors. Participants rate how frequently they engage in each behavior 
Table 1 DWRT program description and objectives

\begin{tabular}{|c|c|}
\hline Chapter overview & Objectives \\
\hline \multicolumn{2}{|l|}{ 1. Introduction } \\
\hline $\begin{array}{l}\text { Participants are engaged in a discussion of the differences between } \\
\text { natural and human caused disasters, stress, and resilience }\end{array}$ & $\begin{array}{l}\text { 1. Defining resilience } \\
\text { 2. Identifying symptoms of acute stress } \\
\text { 3. Deciding when to seek help for psychological symptoms }\end{array}$ \\
\hline \multicolumn{2}{|l|}{ 2. Understanding stress } \\
\hline $\begin{array}{l}\text { Participants learn to define acute and chronic stress and the symptoms } \\
\text { of PTSD. They learn about individual and community level impacts of } \\
\text { disasters }\end{array}$ & $\begin{array}{l}\text { 1. Identifying cognitive, emotional, physical, and social reactions to } \\
\text { f stress } \\
\text { 2. Understanding recovery from stress } \\
\text { 3. Developing awareness of health, safety, and fatigue risk factors }\end{array}$ \\
\hline \multicolumn{2}{|l|}{ 3. What helps? } \\
\hline $\begin{array}{l}\text { Participants learn about factors that help build resilience. They also } \\
\text { learn about the importance of social support and reaching out for } \\
\text { professional mental health care when needed }\end{array}$ & $\begin{array}{l}\text { 1. Learning about the importance of health behaviors (e.g., recuperative } \\
\text { sleep, nutrition, exercise, etc.) } \\
\text { 2. Understanding the importance of social support } \\
\text { 3. Identifying ways to connect with others } \\
\text { 4. Accessing mental health care }\end{array}$ \\
\hline \multicolumn{2}{|l|}{ 4. Resilience and traumatic stress: what is to be done? } \\
\hline $\begin{array}{l}\text { Motivational interviewing informed techniques are used to engage } \\
\text { participants in self-assessment and goal setting. Participants also learn } \\
\text { simple relaxation techniques }\end{array}$ & $\begin{array}{l}\text { 1. Self-assessment of health behaviors } \\
\text { 2. Setting personal health behavior goals } \\
\text { 3. Using relaxation techniques-e.g., deep breathing and visualization } \\
\text { 4. Planning for increasing personal resilience at both an individual and } \\
\text { organizational level }\end{array}$ \\
\hline
\end{tabular}

Chapter content may be modified to address agency specific environmental exposures or working conditions

on a 4-point scale ranging from 1 (Never) to 4 (Routinely). The measure contains six subscales (Health Responsibility, Physical Activity, Nutrition, Spiritual Growth, Interpersonal Relations, and Stress Management). The HPLP II demonstrated excellent reliability in the current sample (total score $\alpha$ 's $=.96$; subscales $\alpha$ 's $=.82-90$ ).

\section{Perceived stress scale (PSS)}

Perceived stress was assessed using the PSS (Cohen et al. 1983), a widely used 10-item self-report questionnaire. Items are rated on a 5-point scale based on how often participants experience specific thoughts, feelings, or difficulties related to stress over the past month, with higher scores indicating more difficulties. The PSS exhibited good reliability in the current study $(\alpha$ 's $=.88-.91)$.

\section{PTSD checklist for DSM-5 (PCL-5)*}

The PCL-5 (Blevins et al. 2015) is a 20-item self-report questionnaire that assesses the severity of each of the DSM-5 criteria for PTSD. Items were anchored to hurricane sandy related exposures. Each item is rated based on how much that symptom had bothered them over the past month on a 5-point Likert-type scale ranging from 0 (Not at all) to 4 (Extremely). It demonstrated excellent reliability in the current sample $(\alpha$ 's $=.92-.94)$.

\section{Patient health questionnaire (PHQ-9)}

The PHQ-9 (Kroenke et al. 2001) is a 10-item self-report measure used to assess the nine DSM- 5 criteria for MDD, with one additional question assessing impairment. Each item is rated on a 4-point scale measuring based on the frequency of the symptom ranging from 0 (Not at all) to 3 (nearly every day). The PHQ-9 showed excellent internal consistency in the current sample ( $\alpha$ 's $=.89-.90)$.

\section{Life events checklist for DSM-5 (LEC-5)}

The LEC-5 (Weathers et al. 2013) is a self-report measure designed to assess exposure to 16 categories of potentially traumatic events. The Life Events Checklist for DSM-IV has good convergent validity with other assessments of traumatic exposures (Gray et al. 2004).

\section{Data analysis}

Where appropriate, independent-samples $t$-tests and Pearson's Chi squared were used to test for differences between the program and waitlist groups on relevant demographic and outcome variables. Race (Caucasian vs. other), marital status (married/cohabitating vs. other) and employment (working/retired vs. disabled/unemployed) were all dichotomized in order to more effectively evaluate their relationship 
to the outcome variables. All analyses were conducted in IBM SPSS Statistics Version 24.

To examine the effect of the DWRT on healthy lifestyle behavior changes, we created a series of Mixed One-way Analysis of Variance (ANOVA) models that analyzed the main effects of condition, time, and the condition by time interaction. Specifically, we tested the hypothesis that participants assigned to the DWRT workshop would have significant improvement in their healthy lifestyle behaviors from $\mathrm{T} 1$ to $\mathrm{T} 2$ relative to the waitlist group. We also ran three additional Mixed ANOVAs in order to assess the effect of the workshop participation on participant perceived stress, PTSD, and depression symptoms.

Finally, in an effort to evaluate the DWRT as a preventative program, we identified participants who experienced subsequent trauma exposures between $\mathrm{T} 1$ and $\mathrm{T} 2$ on the LEC-5. Any participant who answered, "Happened to me," "witnessed it," or "part of my job" for any of the 16 life events or the additional item assessing other extraordinarily stressful life events was included in these analyses. Using this subset of the sample, we reran each of the original models.

\section{Results}

\section{Sample characteristics and descriptive data}

Full sample demographic characteristics are presented in Table 2. There were no significant differences on any of the demographic or outcome variables at $\mathrm{T} 1$ based on condition assignments and thus, in order to conserve power, demographic variables were not retained as covariates in subsequent models.

\section{ANOVA models: complete sample}

ANOVA models were created to analyze the effects of participating in the DWRT workshop on a series of outcome measures. Results for all ANOVA models including the complete sample, as well as descriptive information for all outcome measures at both time points for each condition, are presented in Table 3. We elected not to adjust for multiple comparisons in the present study given that post hoc adjustments are often arbitrary and overly strict, particularly in preliminary and exploratory studies where the risk of failing to detect clinically and statistically significant effects due to the inflation of type 2 error outweigh the risk of identifying potentially spurious effects due to inflation of type 1 error (Althouse 2016; Feise 2002; Rothman 1990).

\section{Healthy lifestyle models}

There was a significant condition by time interaction, such that subjects assigned to the DWRT condition had significantly greater increases in their overall self-reported healthy lifestyle behaviors from $\mathrm{T} 1$ to $\mathrm{T} 2$ than those assigned to the waitlist. Additionally, significant interactions indicate improvements in Physical Activity, Spiritual Growth, and Stress Management, such that the DWRT group improved significantly more from $\mathrm{T} 1$ to $\mathrm{T} 2$ on these measures than those assigned to the waitlist.

\section{Perceived stress, PTSD, and depression models}

Results suggest that there were not any significant effects of condition, time, or the condition by time interactions on Perceived Stress, PTSD symptoms, or depression symptoms.

\section{ANOVA models: trauma-exposed sample}

Of the 167 participants, $101\left(n_{\text {DWRT }}=46, n_{\text {waitlist }}=55\right)$ reported exposure to trauma between their T1 and T2 assessments. The two most commonly reported trauma exposures during the interval were a natural disaster and a transportation accident. The ANOVA models were then recreated to analyze the effect of condition specifically for the subset of the sample who were exposed to a trauma between their baseline and follow-up assessments. Results for all ANOVA models analyzing the trauma-exposed subset participants, as well as descriptive information for all outcome measures at both time points for each intervention group, are presented in Table 4. Plots for selected models demonstrating significant condition by time interactions are presented in Fig. 2.

\section{Healthy lifestyle models}

Among the subset of the sample who reported exposure to trauma between assessments, there was a significant condition by time interaction such that, among subjects exposed to trauma, those assigned to the DWRT workshop improved significantly more from $\mathrm{T} 1$ to $\mathrm{T} 2$ in terms of overall healthy lifestyle habits when compared with the waitlist. Evaluation of the HPLP II subscales indicated that there were significant condition by time interactions for Spiritual Growth and Interpersonal Relations suggesting that among the traumaexposed participants, those assigned to the waitlist condition had significant decreases in these domains relative to individuals assigned to the DWRT. Additionally, there was a significant condition by time interaction for Stress Management demonstrating that, among the trauma-exposed participants, those assigned to the DWRT group reported 
Table 2 Demographic characteristics

\begin{tabular}{|c|c|c|}
\hline Demographic variable & DWRT group $(N=76)$ & Waitlist group $(N=83)$ \\
\hline Age $M(\mathrm{SD})$ & $51.75(15.33)$ & $51.19(15.53)$ \\
\hline \multicolumn{3}{|l|}{ Gender $N(\%)$} \\
\hline Male & $39(51.3 \%)$ & $33(39.8 \%)$ \\
\hline Female & $36(47.4 \%)$ & $49(59.0 \%)$ \\
\hline Prefer not to answer & $1(1.3 \%)$ & $1(1.2 \%)$ \\
\hline \multicolumn{3}{|l|}{ Marital status $N(\%)$} \\
\hline Living with partner & $4(5.3 \%)$ & $11(13.3 \%)$ \\
\hline Divorced & $6(7.9 \%)$ & $5(6.0 \%)$ \\
\hline Married & $43(56.6 \%)$ & $33(39.8 \%)$ \\
\hline Separated & $2(2.6 \%)$ & $3(3.6 \%)$ \\
\hline Single & $18(23.7 \%)$ & $27(32.5 \%)$ \\
\hline Widowed & $1(1.3 \%)$ & $4(4.8 \%)$ \\
\hline Prefer not to answer & $2(2.6 \%)$ & $0(0 \%)$ \\
\hline \multicolumn{3}{|l|}{ Racial group $N(\%)$} \\
\hline American Indian/Alaskan native & $0(0 \%)$ & $1(1.2 \%)$ \\
\hline Asian & $6(7.9 \%)$ & $3(3.6 \%)$ \\
\hline Black/African American & $8(10.5 \%)$ & $13(15.7 \%)$ \\
\hline White & $52(68.4 \%)$ & $60(72.3 \%)$ \\
\hline Other/multi racial & $6(7.9 \%)$ & $3(3.6 \%)$ \\
\hline Prefer not to answer & $4(5.3 \%)$ & $3(3.6 \%)$ \\
\hline \multicolumn{3}{|l|}{ Ethnicity $N(\%)$} \\
\hline Non-hispanic & $65(85.5 \%)$ & $73(88.0 \%)$ \\
\hline Hispanic & $8(10.5 \%)$ & $6(7.2 \%)$ \\
\hline Prefer not to answer & $3(3.9 \%)$ & $4(4.8 \%)$ \\
\hline \multicolumn{3}{|l|}{ Employment $N(\%)$} \\
\hline Paid full time employment & $43(56.6 \%)$ & $38(45.8 \%)$ \\
\hline Paid part time employment & $5(6.6 \%)$ & $15(18.1 \%)$ \\
\hline Unemployed & $5(6.6 \%)$ & $3(3.6 \%)$ \\
\hline Retired & $14(18.4 \%)$ & $21(25.3 \%)$ \\
\hline Disabled & $4(5.2 \%)$ & $3(3.6 \%)$ \\
\hline Prefer not to answer & $5(6.6 \%)$ & $3(3.6 \%)$ \\
\hline \multicolumn{3}{|l|}{ Response to Hurricane Sandy N (\%) } \\
\hline As part of job & $13(17.1 \%)$ & $16(19.3 \%)$ \\
\hline As a volunteer & $49(64.5 \%)$ & $48(57.8 \%)$ \\
\hline Both & $13(17.1 \%)$ & $17(20.5 \%)$ \\
\hline Prefer not to answer & $1(1.3 \%)$ & $2(2.4 \%)$ \\
\hline
\end{tabular}

$D W R T$ disaster worker resiliency training

significant improvement in stress management skills from $\mathrm{T} 1$ to $\mathrm{T} 2$ relative to participants in the waitlist (see Fig. 2a).

\section{Perceived stress model}

The model examining changes in perceived stress among the trauma-exposed subsample showed that there was a significant condition by time interaction demonstrating that, after exposure to trauma, participants assigned to the waitlist group had significant increases in perceived stress from T1 to T2 compared to subjects in the DWRT group (see Fig. 2b).

\section{PTSD model}

Analysis of change in PTSD symptoms in the traumaexposed subsample revealed that there was a significant condition by time interaction, indicating that, following exposure to trauma, participants assigned to the waitlist had a significantly increased levels of PTSD symptoms from T1 to $\mathrm{T} 2$ relative to subjects assigned to the DWRT group (see Fig. 2c). 


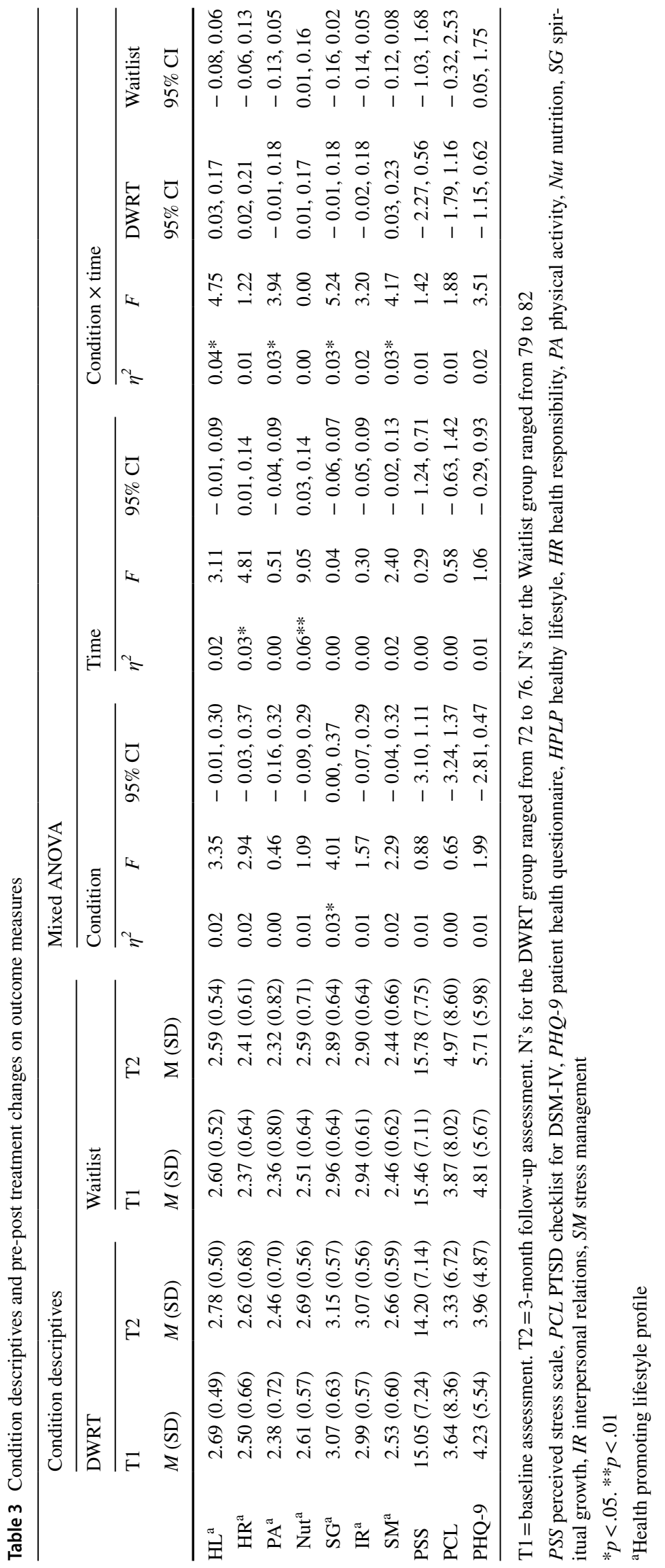




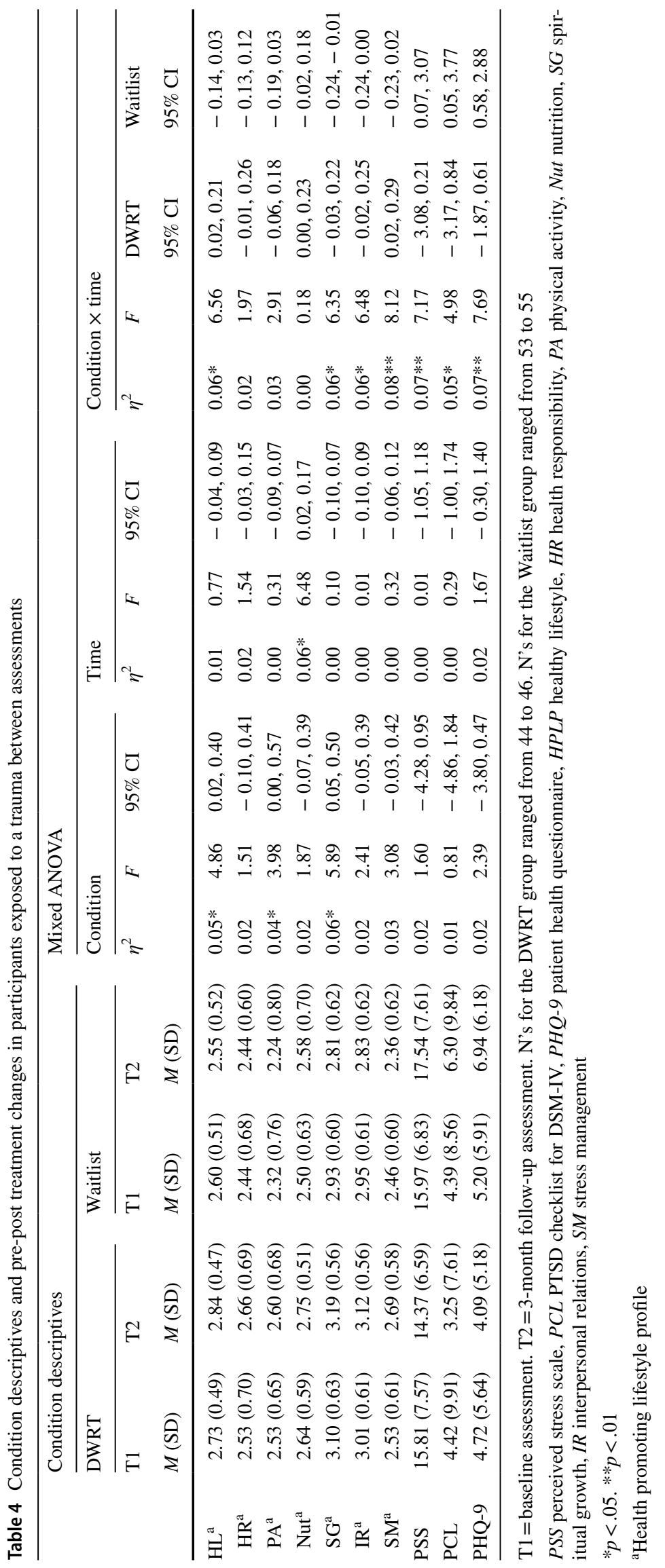




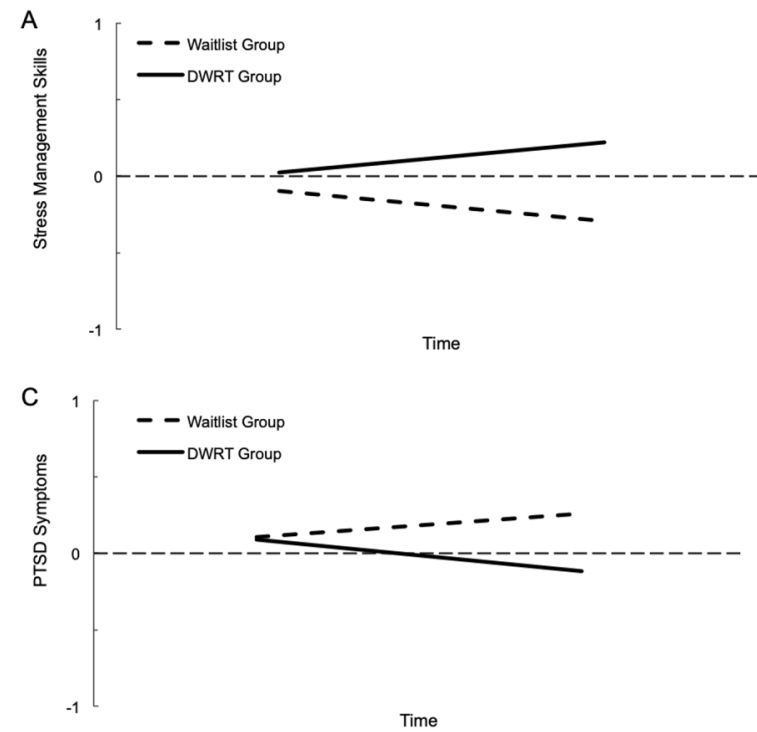

Fig. 2 Trajectories of perceived stress, PTSD, and depression symptoms. This figure illustrates the changes in stress management skills (a), perceived stress (b), PTSD (c), and depression symptoms (d) from baseline (T1) to the 3-month follow-up assessment (T2) DWRT and waitlist groups among participants who experienced a trauma

\section{Depression model}

Similar to the perceived stress and PTSD models, the model investigating depression symptoms among the traumaexposed sample revealed that there was a significant condition by time interaction such that, among trauma-exposed participants, those assigned to the waitlist condition had significantly increased depression symptoms from $\mathrm{T} 1$ to $\mathrm{T} 2$ compared to subjects in the DWRT group (see Fig. 2d).

\section{Discussion}

Disaster workers, particularly those with multiple disaster exposures are at increased risk for health problems and trauma-related mental health problems, such as PTSD and depression. Resilience building interventions, such as the DWRT, may offer responders a means of developing adaptive coping skills and offsetting the negative effects of future disaster exposures (Davydov et al. 2010). The present study sought to test the efficacy of the DWRT in a sample of 167 disaster responders exposed to Hurricane Sandy. The DWRT is unique from other interventions, such as Psychological First Aid and CISM, which were designed for mitigating symptoms after a crisis, rather than building resilience to the mental health effects of disaster work in-advance of a crisis. This is particularly salient in the context of the recent global crisis (COVID-19), which has placed a significant burden on

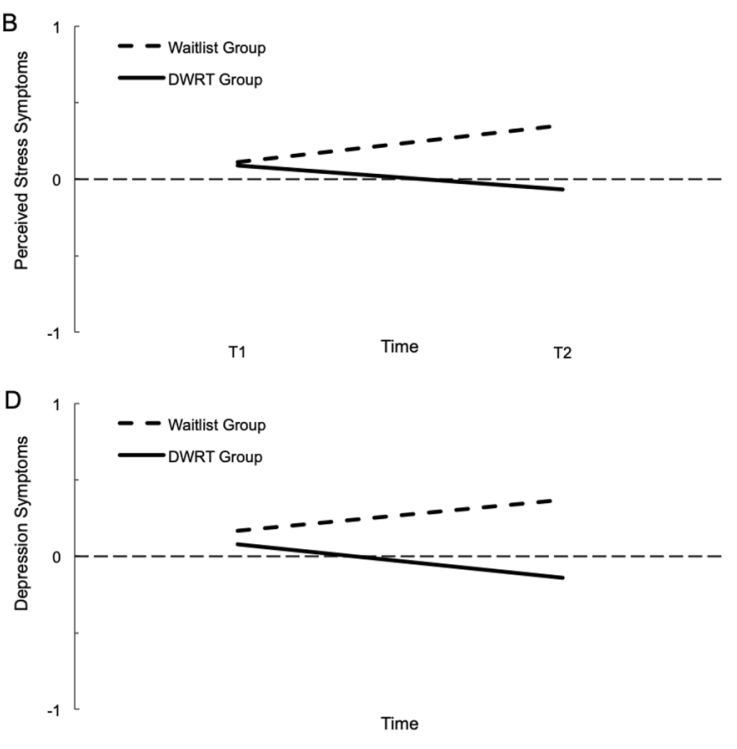

between $\mathrm{T} 1$ and $\mathrm{T} 2$. Outcomes are reported as Z-scores and therefore have a mean of 0 and a standard deviation of $1 . D W R T$ disaster worker resiliency training, PTSD posttraumatic stress disorder, T1 pre-intervention assessment, $T 2$ 2-month follow-up assessment

the mental health of healthcare workers and other front-line responders (Lai et al. 2020).

Results of the investigation suggest that at 3 months postrandomization, those completing the DWRT, as compared to waitlist, reported significantly greater improvements in physical activity, spiritual growth and stress management coping skills. While improvements were modest, they are nonetheless remarkable given the brevity of this intervention and the heightened vulnerability of this population to stress and trauma-related health conditions. Beyond evident physical health benefits (Penedo and Dahn 2005), cardiovascular exercise is known to substantially reduce mental health symptoms such as depression and anxiety (Stathopoulou et al. 2006). Emerging evidence also supports the efficacy of exercise for reducing the severity of PTSD symptoms in children, adolescents and adults (Wolff et al. 2011). A variety of mechanisms by which exercise confers benefits have been proposed including increased social contact, improved self-esteem and biological factors such as increased synthesis and release of neurotransmitters and neurotrophic factors (Portugal et al. 2013). Therefore, it is possible that increasing physical activity in disaster worker populations could increase both physiological and psychosocial resilience. Likewise, spirituality has been linked with overall better quality of life and reduced PTSD in both veteran and trauma-exposed community samples (Connor et al. 2003; Currier et al. 2015). Thus, increased spirituality may also buffer in some ways against the deleterious effects of trauma exposure. 
In the general sample, however, participation in the DWRT program did not confer any benefit in terms of Sandy-related PTSD symptoms, perceived stress or depression symptoms. This may be for a number of reasons including overall low base rates of these symptoms in the present sample. Although we selected participants on the basis of their engagement in either voluntary or job-related disaster response efforts to Hurricane Sandy, many participants primarily reported work-related exposures and reported low levels of personal storm-related exposures such as property loss/damage, financial hardship, or physical injuries. This is notable as personal losses and physical injuries are important risk factors for the development of PTSD (Norris et al. 2010). Moreover, around $40 \%$ of the overall sample denied experiencing additional trauma exposure in the interceding 3 months between randomization and the follow-up assessment.

Thus, in order to examine the impact of participation in the DWRT program on resilience to future stressors, we selected a subset of the sample that endorsed exposure to at least one traumatic event between $\mathrm{T} 1$ and $\mathrm{T} 2$. In this sub-sample, time by condition interactions were observed wherein those who participated in the DWRT were significantly likely to report improved stress-management skills from T1 to T2 and levels of perceived stress, depression and PTSD symptoms either stayed the same or decreased from $\mathrm{T} 1$ to $\mathrm{T} 2$. However, trauma-exposed participants in the waitlist condition experienced a significant increase in mental health symptoms. This suggests that participation in the DWRT engaged the target of teaching adaptive coping skills and conferred resilience to subsequent trauma exposures, which was not observed in the waitlist group. Again, while effect sizes were modest, even small improvements in health behaviors, self-care, and resistance to trauma-related psychological symptoms may foster a more dynamic workforce that is able to continue responding to long-lasting crises, such as the recent COVID-19 pandemic.

While this represents a notable first step towards developing evidence-based resilience building programing for disaster workers, the present study also has limitations, which should be addressed in future work. First, the present sample was heterogeneous in terms of occupation and volunteer versus professional responder status. As such, degree of disaster exposure, amount of formal disaster response training, and risk for subsequent disaster/trauma exposures likely varied substantially across the sample. Thus, additional research is needed in larger samples to enable exploration of potential moderators of intervention efficacy. Further, randomization was done within agencies, thus it is possible that social transmission of intervention information happened between active and waitlist participants. This may have attenuated outcomes in the intervention group. A cluster randomization strategy may be used to address this in future studies. Overall rates of PTSD and depression symptoms were also relatively low in the present sample. Thus, it remains unknown how well this intervention may work in samples with higher levels of stress, psychopathology, or burnout. Moreover, mental health symptoms and health behaviors were assessed via self-report instruments. Future work would be strengthened by the incorporation of multimethod outcome assessment. For the current study, the DWRT workshops were delivered by trained mental health professionals. Mental health professionals are not always readily available in disaster settings and training workers within agencies to deliver the intervention may promote its dissemination. Thus, future research should assess the efficacy of the DWRT as delivered by trained peers. It also remains unknown how treatment expectancy effects affected outcomes. Future work should compare the DWRT to active control conditions, matched for time and trainer-attention. Finally, the sample was only followed through 3-months post randomization and, as such, it remains unknown how durable the effects of the intervention will be. Future studies should incorporate additional follow ups to address the question of whether follow-up or booster trainings are necessary to sustain the effects of resilience-building interventions.

In sum, the present study is the first RCT to evaluate the efficacy of the DWRT, a brief preventative intervention for resilience training in active disaster responders. The DWRT is a manualized resilience building intervention that is easy to disseminate, and appropriate for a wide variety of disaster worker groups. Preliminary evidence from NIEHS Grantee communities, such as groups affected by Hurricane Maria in Puerto Rico, suggests that peer trainer models may also be feasible and increase the potential reach of the intervention. While previous disaster mental health interventions have focused on post-trauma exposures (e.g., psychological first aid and CISM), limited systematic efforts have focused on the development and testing of targeted preventative interventions for promoting resilience in poly-trauma exposed populations. Continued efforts are needed to ensure the resilience of the disaster response workforce; efforts vital to maintaining national security and the safety of communities.

Acknowledgements We would like to thank Mitchel Rosen, $\mathrm{PhD}$ for aiding in recruitment; Philip McCabe, MS for serving as a workshop instructor; Vincenza Caruso for serving as the project coordinator; and Lucero Molina for assisting with editing this manuscript.

Funding This study was supported by a grant from the Assistant Secretary for Preparedness and Response (ASPR), US Department of Health and Human Services (DHHS; 1-HITEP-140021-01-00) awarded to A.G. 


\section{Compliance and ethical standards}

Conflict of interest The authors declare they have no actual or potential competing financial interests.

Informed consent This study was approved by the institutional review board of Stony Brook University. All participants provided informed consent prior to participating in this study.

\section{References}

Althouse AD (2016) Adjust for multiple comparisons? It's not that simple. Ann Thorac Surg 101(5):1644-1645

Benedek DM, Fullerton C, Ursano RJ (2007) First responders: mental health consequences of natural and human-made disasters for public health and public safety workers. Annu Rev Public Health 28:55-68. https://doi.org/10.1146/annurev.publhealth.28.02140 6.144037

Berger W, Coutinho ES, Figueira I, Margues-Portella C, Luz MP, Neylan TC, Marmar CR, Mendlowicz MV (2012) Rescuers at risk: a systematic review and meta-regression analysis of the worldwide current prevalence and correlates of PTSD in rescue workers. Soc Psychiatry Psychiatr Epidemiol 47(6):1001-1011. https:// doi.org/10.1007/s00127-011-0408-2

Blevins CA, Weathers FW, Davis MT, Witte TK, Domino JL (2015) The posttraumatic stress disorder checklist for DSM-5 (PCL-5): development and initial psychometric evaluation. J Trauma Stress 28(6):489-498

Breslau N, Peterson EL, Schultz LR (2008) A second look at prior trauma and the posttraumatic stress disorder effects of subsequent trauma: a prospective epidemiological study. Arch Gen Psychiatry 65(4):431-437. https://doi.org/10.1001/archpsyc.65.4.431

Bromet EJ, Atwoli L, Kawakami N, Navarro-Mateu F, Piotrowski P, King AJ, Aguilar-Gaxiola S, Alonso J, Bunting B, Demyttenaere K, Florescu S (2017a) Post-traumatic stress disorder associated with natural and human-made disasters in the World Mental Health Surveys. Psychol Med 47(2):227-241. https://doi. org/10.1017/S0033291716002026

Bromet EJ, Clouston S, Gonzalez A, Kotov R, Guerrera KM, Luft BJ (2017b) Hurricane sandy exposure and the mental health of world trade center responders. J Trauma Stress 30(2):107-114. https:// doi.org/10.1002/jts.22178

Bruce ML, Leaf PJ, Rozal GPM, Floria L, Hoff RA (1994) Psychiatric status and 9-year mortality data in the New Haven Epidemiologic Catchment Area Study. Am J Psychiatry 151(5):716. https://doi. org/10.1176/ajp.151.5.716

Brunet A, Boyer R, Weiss DS, Marmar CR (2001) The effects of initial trauma exposure on the symptomatic response to a subsequent trauma. Can J Behav Sci 33(2):97-102. https://doi.org/10.1037/ h0087132

Caramanica K, Brackbill RM, Stellman SD, Farfel MR (2015) Posttraumatic stress disorder after Hurricane Sandy among persons exposed to the 9/11 disaster. Int J Emerg Ment Health 17(1):356

Cohen S, Kamarck T, Mermelstein R (1983) A global measure of perceived stress. J Health Soc Behav 385-396

Connor KM, Davidson JRT, Lee LC (2003) Spirituality, resilience, and anger in survivors of violent trauma: a community survey. $\mathrm{J}$ Trauma Stress 16(5):487-494. https://doi.org/10.1023/A:10257 62512279

Currier JM, Holland JM, Drescher KD (2015) Spirituality factors in the prediction of outcomes of PTSD treatment for US military veterans. J Trauma Stress 28(1):57-64
Davydov DM, Stewart R, Ritchie K, Chaudieu I (2010) Resilience and mental health. Clin Psychol Rev 30(5):479-495. https://doi. org/10.1016/j.cpr.2010.03.003

Feise RJ (2002) Do multiple outcome measures require p-value adjustment?. BMC Med Res Methodol 2(1):8

Fox JH, Burkle FM, Bass J, Pia FA, Epstein JL, Markenson D (2012) The effectiveness of psychological first aid as a disaster intervention tool: research analysis of peer-reviewed literature from 1990-2010. Disaster Med Public Health Prep 6(3):247-252

Galea S, Nandi A, Vlahov D (2005) The epidemiology of post-traumatic stress disorder after disasters. Epidemiol Rev 27:78-91. https://doi.org/10.1093/epirev/mxi003

Gray MJ, Litz BT, Hsu JL, Lombardo TW (2004) Psychometric properties of the life events checklist. Assessment 11(4):330-341

Irving P, Long A (2001) Critical incident stress debriefing following traumatic life experiences. J Psychiatr Ment Health Nurs 8(4):307-314. https://doi.org/10.1046/j.1351-0126.2000.00388 .X

Kabat-Zinn J (2003) Mindfulness-based stress reduction (MBSR). Constr Hum Sci 8(2):73

Kroenke K, Spitzer RL, Williams JB (2001) The PHQ-9: validity of a brief depression severity measure. J Gen Intern Med 16(9):606-613

Lai J, Ma S, Wang Y, Cai Z, Hu J, Wei N, Wu J, Du H, Chen T, Li R, Tan H (2020) Factors associated with mental health outcomes among health care workers exposed to coronavirus disease 2019 . JAMA Netw Open 3(3):e203976-e203976

Lavretsky H, Bastani R, Gould R, Huang D, Llorente M, Maxwell A, Jarvik L (2002) Predictors of two-year mortality in a prospective "UPBEAT" study of elderly veterans with comorbid medical and psychiatric symptoms. Am J Geriatr Psychiatry 10(4):458-468. https://doi.org/10.1097/00019442-200207000-00012

Mitchell JT, Everly GS (1997a) Critical incident stress debriefing (CISD): an operations manual for the prevention of traumatic stress among emergency service and disaster workers, revised, 2nd edn. Chevron Publishing Corporation, Ellicott City

Mitchell JT, Everly GS (1997b) The scientific evidence for critical incident stress management. JEMS 22(1):86-93

Nash WP, Watson PJ (2012) Review of VA/DOD clinical practice guideline on management of acute stress and interventions to prevent posttraumatic stress disorder. J Rehabil Res Dev 49(5):637-648

National Institute for Environmental Health Sciences (2012) Responder and community resilience: training resources. https://tools.niehs .nih.gov/wetp/index.cfm?id=2528

Neria Y, Nandi A, Galea S (2008) Post-traumatic stress disorder following disasters: a systematic review. Psychol Med 38(4):467-480. https://doi.org/10.1017/s0033291707001353

New York State Department of Health (2012) Health advisory: respiratory health and related illness in areas impacted by Hurricane Sandy. http://www.health.ny.gov/environmental/emergency/weath er/hurricane/docs/2012-12-05_health_advisory_respiratory_healt h_letter.pdf. Accessed 20 Jan 2013

Norris FH, Sherrieb K, Galea S (2010) Prevalence and consequences of disaster-related illness and injury from Hurricane Ike. Rehabil Psychol 55(3):221

Park ER, Traeger L, Vranceanu AM, Scult M, Lerner JA, Benson H, Denninger J, Fricchione GL (2013) The development of a patientcentered program based on the relaxation response: the Relaxation Response Resiliency Program (3RP). Psychosomatics 54(2):165174. https://doi.org/10.1016/j.psym.2012.09.001

Penedo FJ, Dahn JR (2005) Exercise and well-being: a review of mental and physical health benefits associated with physical activity. Curr Opin Psychiatry 18(2):189-193

Portugal EM, Cevada T, Monteiro-Junior RS, Guimarães TT, da Cruz Rubini E, Lattari E, Blois C, Deslandes AC (2013) Neuroscience 
of exercise: from neurobiology mechanisms to mental health. Neuropsychobiology 68(1):1-14

Rothman KJ (1990) No adjustments are needed for multiple comparisons. Epidemiology 43-46

Ruzek JI, Brymer MJ, Jacobs AK, Layne CM, Vernberg EM, Watson PJ (2007) Psychological first aid. J Ment Health Couns 29(1):17-49

Shrira A, Palgi Y, Hamama-Raz Y, Goodwin R, Ben-Ezra M (2014) Previous exposure to the World Trade Center terrorist attack and posttraumatic symptoms among older adults following Hurricane Sandy. Psychiatry 77(4):374-385

Sood A, Prasad K, Schroeder D, Varkey P (2011) Stress management and resilience training among Department of Medicine faculty: a pilot randomized clinical trial. J Gen Intern Med 26(8):858-861. https://doi.org/10.1007/s11606-011-1640-x

Stathopoulou G, Powers MB, Berry AC, Smits JA, Otto MW (2006) Exercise interventions for mental health: a quantitative and qualitative review. Clin Psychol Sci Pract 13(2):179-193

Thormar SB, Gersons BP, Juen B, Marschang A, Djakababa MN, Olff M (2010) The mental health impact of volunteering in a disaster setting: a review. J Nerv Ment Dis 198(8):529-538. https://doi. org/10.1097/NMD.0b013e3181ea1fa9
Tuckey MR, Scott JE (2014) Group critical incident stress debriefing with emergency services personnel: a randomized controlled trial. Anxiety Stress Coping 27(1):38-54

Walker SN, Sechrist KR, Pender NJ (1995) Health promotion modelinstruments to measure health promoting lifestyle: health-promoting lifestyle profile [HPLP II] (Adult version)

Weathers FW, Blake DD, Schnurr PP, Kaloupek DG, Marx BP, Keane TM (2013) The Life Events Checklist for DSM-5 (LEC-5)

White House (2007) Homeland Security Presidential Directive/ HSPD-21

Wolff E, Gaudlitz K, von Lindenberger BL, Plag J, Heinz A, Ströhle A (2011) Exercise and physical activity in mental disorders. Eur Arch Psychiatry Clin Neurosci 261(2):186

Publisher's Note Springer Nature remains neutral with regard to jurisdictional claims in published maps and institutional affiliations. 